\title{
Franz Knipping \\ Die Römischen Verträge von 1957: eine nachhaltige Grundlegung
}

In der Jubiläumskultur der Europäischen Union erfährt inzwischen kaum ein anderes Ereignis eine so hohe Aufmerksamkeit wie die Unterzeichnung der Verträge zur Gründung der Europäischen Wirtschaftsgemeinschaft und zur Gründung der Europäischen Atomgemeinschaft am 25. März 1957 auf dem Kapitolshügel in Rom. Nur die Verkündung des SchumanPlans am 9. Mai 1950, inzwischen auch Referenzdatum für den alljährlichen „Europatag“, findet vergleichbare Beachtung. Die Zehnjahrestage der Römischen Verträge wurden seit 1967 von den europäischen Institutionen und den Regierungen der Mitgliedstaaten mit zunehmendem Aufwand feierlich begangen, und seit dem 30. Jahrestag 1987 bieten sie auch besonderen Anlass, sich mit der Geschichte der europäischen Einigung aus der Sicht von Zeitzeugen und Historikern intensiver zu beschäftigen, durchaus auch in identitätsstiftender Absicht. Sowohl 1987 als auch 1997 fanden neben den offiziellen Feierlichkeiten jeweils Veranstaltungen und wissenschaftliche Tagungen statt, insbesondere bemerkenswerte Kolloquien an der Stätte der Unterzeichnung der Verträge. Der 40. Jahrestag 1997 führte 39 führende europäische Akteure, aktive und ehemalige, mit 45 Historikern der europäischen Einigung zusammen, die die Umsetzung der Gründungsverträge und die von ihnen ausgehende Entwicklungsdynamik zu bilanzieren versuchten. Dabei hielt Leo Tindemans zusammenfassend fest: „Der große Augenblick [der Europäischen Integration] waren offenkundig die Verträge von Rom, die uns auch weiter inspirieren müssen“.

Zum 50. Jahrestag blickte der Europäische Rat der Staats-und Regierungschefs dann in einer „,Berliner Erklärung“ auf die einzigartige Erfolgsgeschichte der Union zurück: „Europa war über Jahrhunderte eine Idee, eine Hoffnung auf Frieden und Verständigung. Diese Hoffnung hat sich erfüllt. Die europäische Einigung hat uns Frieden und Wohlstand ermöglicht. Sie hat Gemeinsamkeit gestiftet und Gegensätze überwunden. Jedes Mitglied hat geholfen, Europa zu einigen und Demokratie und Rechtsstaatlichkeit zu stärken. Der Freiheitsliebe der Menschen in Mittel- und Osteuropa verdanken wir, dass heute Europas unnatürliche Teilung endgültig überwunden ist. Wir haben mit der europäischen Einigung unsere Lehren aus blutigen Auseinandersetzungen und leidvoller Geschichte gezogen. Wir leben heute miteinander, wie es nie zuvor möglich war. Wir Bürgerinnen und Bürger der Europäischen Union sind zu unserem Glück vereint“. Zur gleichen Zeit fand auf dem Kapitol wieder ein internationales, diesmal eher akademisches und interdisziplinäres Kolloquium statt, das die „50 years of European Construction 1957-2007“ im Licht der neueren Forschungen Revue passieren ließ.

Damals, am 25. März 1957, war den Akteuren, Gästen und Medienvertretern auf dem Kapitol völlig bewusst, dass sie einer europäischen „Sternstunde“ beiwohnten und Geschichte schrieben. Die Delegationen der sechs Vertragsstaaten leiteten den historischen Akt mit der Teilnahme an einer feierlichen Messe in S. Paolo fuori le Mura zum Gedenken an den drei Jahre zuvor verstorbenen Alcide De Gasperi ein. Papst Pius XII. persönlich veranlasste offenbar, dass danach der Autokonvoi zum Kapitol vom Geläut aller Glocken Roms begleitet wurde. Um 18 Uhr begann im Konservatorenpalast, wo für die Regierungsvertreter ein zwölf Meter langer Tisch aufgebaut war, in Anwesenheit von mehreren Hundert Beteiligten die knapp einstündige Unterzeichnungszeremonie, eingeleitet von kurzen Ansprachen. Der italienische Außenminister Martino sprach vom „Beginn einer neuen Ära in der Geschichte 
der europäischen Völker“. Bundeskanzler Adenauer beschwor den genius loci: „Europa hätte keinen würdigeren Rahmen für diese Konferenz finden können als diese seine ehrwürdigste Stadt“. Die Unterzeichnung der Verträge bedeute einen ,geschichtlichen Augenblick. Wir wollen uns sicherlich nicht Vorschusslorbeeren winden. Allzuviel an Aufgaben liegt vor uns. Aber die Freude darüber, dass es uns vergönnt ist, den großen Schritt zur Einigung Europas zu tun, der in der Unterzeichnung der Verträge liegt - dieser Freude möchte ich doch Ausdruck geben. Die Optimisten, nicht die Pessimisten haben recht behalten“. Der belgische Außenminister Spaak pries den Mut der Europäer, aus freien Stücken die größte Umwälzung ihrer Geschichte in Angriff zu nehmen. „Wenn wir das begonnene Werk fortsetzen, wird der Tag des 25. März 1957 einer der bedeutendsten in der Geschichte Europas sein“. Mit kaum geringerem Pathos äußerten sich der französische Außenminister Pineau und der niederländische Außenminister Luns. Der luxemburgische Staats- und Außenminister Bech schließlich beschwor den Geist Europas. Es werde lange dauern, bis das vereinte Europa vollständig errichtet sei, doch werde ständige Zusammenarbeit die Solidarität der Völker entwickeln. „Ceterum censeo Europam esse construendam“. Die Unterschriften unter die Verträge wurden danach mit von der Stadt Rom gestifteten goldenen Füllfederhaltern geleistet, während die Glocken des Kapitols zu läuten begannen, darunter die über 700 Jahre alte Patarina. In den folgenden Stunden wurde bei einem Bankett in der Villa Madama und einem Empfang im Palazzo Venezia das historische Ereignis in Anwesenheit von mehr als tausend geladenen Gästen ausgiebig gefeiert.

Auch die Medien wussten die Geschichtlichkeit des Augenblicks zu würdigen. Fast alle Zeitungen des kontinentalen Westeuropa berichteten am 26. und 27. März in großer Aufmachung über den historischen Akt in Rom, so gut wie ausnahmslos in lebhaft zustimmender Weise. Die Berichterstattung in Großbritannien und den skandinavischen Ländern war etwas zurückhaltender, hier wurden auch konkurrierende Topmeldungen des Tages stärker herausgestellt. Unverhohlene Reserve zeigten nur die kommunistischen Blätter. Ganz überwiegend feierten aber die westeuropäischen Medien den „Beginn eines neuen Abschnitts der Geschichte Europas“. Es wurde sogar, bedeutungsschwanger, nachgerechnet, dass am 25. März 1957 exakt 2000 Jahre seit der Ermordung Cäsars vergangen waren. Auch von jenseits des Atlantik kam Zustimmung. Die amerikanische Presse variierte die Verlautbarung des Washingtoner Außenministeriums, dass die Europäer eine begrüßenswerte Initiative ergriffen hätten und die Römischen Verträge einen Meilenstein auf dem Weg der Integration Europas darstellten.

Es ist allerdings einzuräumen, dass die positive Resonanz der veröffentlichten Meinung der Aufnahme in der breiten Bevölkerung vielleicht nicht in Gänze entsprach. In Italien war immerhin den Schulkindern frei gegeben worden. Doch insgesamt scheint die Aura des Tages in Rom den Alltag der Europäer nicht wirklich überstrahlt zu haben. Europäische Wirtschafts- und Atomgemeinschaft waren neue, komplexe Vorhaben, die nach dem Scheitern der Europäischen Verteidigungsgemeinschaft 1954 schlecht einzuordnen waren. Gewiss war die Grundstimmung positiv, doch manches zeitgenössische Zeugnis offenbart, dass die Menschen auf die Nachrichten aus Rom insgesamt eher gleichgültig reagierten. Eine gewisse Distanz zwischen gouvernementaler Aktion und demokratischer Partizipation im europäischen Einigungsprozess deutete sich schon hier an: die europäische Einigung zuvorderst als Projekt der politischen (und wirtschaftlichen) Führungen.

Für die sachliche Bewertung erscheint rückschauend der Befund eindeutig. Mit dem Akt auf dem Kapitol wurde eine der positivsten und konstruktivsten politischen Entwicklungen eingeleitet, die das an Gewalttätigkeiten überreiche 20. Jahrhundert hervorgebracht hat. Markierte der Schuman-Plan die „Geburtsstunde“ der europäischen Integration, so wurden die Römischen Verträge zu ihrer eigentlichen Grundlegung. Die Römischen Verträge waren der 
eigentliche Durchbruch, sie schufen den festen Rahmen, stießen die Tür weit auf zu einer zukunftsfähigen Entwicklung, wie sie, ungeachtet immer wiederkehrender Krisen, in der gegenwärtigen Gestalt der Europäischen Union weit vorangeschritten vor uns steht.

Dabei übernahm der EWG-Vertrag eine Leitfunktion. Er mutierte zu dem roten Faden, an dem entlang sich bis heute die Dynamik des Integrationsprozesses entfaltet hat. Euratom wie Montanunion machten sich damals daran, einzelne Sektoren der Wirtschaften der Mitgliedstaaten zu vergemeinschaften, aber beide gerieten schon in den 1960er Jahren in schweres Fahrwasser. Euratom versank zwischen den nuklearindustriellen Individualinteressen der Mitgliedstaaten schon seit den 1960er Jahren in Bedeutungslosigkeit. Die Montanunion litt alsbald unter Absatzkrisen von Kohle und Stahl und ist inzwischen sogar, nach 50 Jahren des Bestehens, seit 2002 aufgelöst. Die Europäische Wirtschaftsgemeinschaft wurde dagegen zum eigentlichen Kern des Europaprojekts. Sie hatte nicht einzelne Sektoren der Wirtschaft, sondern die Vergemeinschaftung der Wirtschaften der Mitgliedstaaten insgesamt im Visier. Ihre Gründung bedeutete den Sieg der Idee einer „,horizontalen“ Integration, wie sie vor allem der niederländische Außenminister Johan Willem Beyen propagierte, über das Konzept der „sektoralen“ Integration, das Jean Monnet bevorzugte. Das Ziel, so hatte Beyen seit 1952 argumentiert, müsse ein umfassender gemeinsamer Markt sein, sein Kern eine Zollunion mit stufenweisem Abbau der Binnenzölle und gemeinsamem Außenzoll. „Jede Teilintegration hat die Tendenz, Schwierigkeiten in einem Sektor durch Maßnahmen zu lösen, die andere Sektoren oder die Interessen der Verbraucher beeinträchtigen und zum Ausschluss der ausländischen Konkurrenz führen. Dies ist nicht der Weg zur Erhöhung der europäischen Produktivität. Außerdem trägt die sektorale Integration nicht im gleichen Maße zur Stärkung des Gefühls der Solidarität und Einheit Europas bei wie eine gesamtwirtschaftliche Integration“. Diese Auffassung setzte sich durch. Der umfassende EWG-Vertrag wurde zur Basis für alles andere. Er stellte die innere Sachlogik des integrativen Fortschreitens bereit, die in mehrfacher Ergänzung des Vertragstextes über die Fusion der Exekutiven, die Schaffung eines europäischen Marktes und einer gemeinsamen Währung, die Erweiterungsrunden, die Ausweitung des Mehrheitsvotums im Ministerrat und die Zielsetzung einer gemeinsamen Innen-, Außen- und Verteidigungspolitik der Politischen Union zustrebte. Der MaastrichtVertrag, der die Europäische Union schuf, inkorporierte den schon durch die Einheitliche Europäische Akte (1987) fortgeschriebenen EWG-Vertrag als EG-Vertrag, der Vertrag von Lissabon übernahm ihn als „Vertrag über die Arbeitsweise der Union“ (AEUV).

Im Rückblick ist unübersehbar, dass der EWG-Vertrag von Rom das wesentliche Instrument, den Motor für die Schaffung eines großen europäischen Wirtschafts- und Verkehrsraums bereitstellte, wie es ihn in der europäischen Geschichte bisher noch nicht gegeben hat. Zunächst schuf er die Zollunion der sechs Gründerstaaten für gewerbliche Güter, und zwar rascher als vorgesehen. Die in Artikel 8 des EWG-Vertrags vorgesehene Übergangszeit von 12 bis 15 Jahren brauchte angesichts einer günstigen Wirtschafts- und Handelsentwicklung zwischen den sechs Gründerstaaten nicht ausgeschöpft zu werden. Schon nach zehneinhalb Jahren, am 1. Juli 1968, waren die Zölle und mengenmäßigen Beschränkungen zwischen ihnen vollständig aufgehoben und ein gemeinsamer Außentarif vereinbart. Die gleichzeitige Zollunion auch für landwirtschaftliche Güter im Rahmen der Gemeinsamen Agrarpolitik, die 1970 abgeschlossen wurde, komplettierte den „Gemeinsamen Markt“, der strukturelle Optimierungen und zusätzlichen Wachstumsschub ermöglichte, auch eine Verminderung der Produktionskosten und Preisunterschiede und letztlich eine fühlbare Erhöhung der Kaufkraft der Verbraucher.

Derselbe Funktionalismus des EWG-Vertrags entfaltete seit den 1970er Jahren die Dynamik für die Fortentwicklung des Integrationsprozesses im Sinn einer stetigen Erweiterung und Vertiefung. Der große europäische Wirtschafts- und Verkehrsraum vergrößerte sich 
1973 um die nördlichen Länder Großbritannien, Irland und Dänemark, dann in den 1980er Jahren um die Südländer Griechenland, Portugal und Spanien. 1995 traten Finnland, Schweden und Österreich hinzu, und die Großerweiterung der Jahre 2004 und 2007, um insgesamt zehn osteuropäische Länder und die Inselstaaten Malta und Zypern, vermehrte die Zahl der Mitgliedstaaten auf nunmehr 27. Entstanden ist über die Jahrzehnte ein imposanter Großraum von rund 4,3 Millionen Quadratkilometern mit etwa 500 Millionen Einwohnern, der vom Volumen her zweitgrößte Wirtschaftsraum der Welt (nach den USA) und zugleich die größte Handelsmacht, größter Exporteur und zweitgrößter Importeur.

Aber auch die Vertiefung verstetigte sich und bewirkte verlässliche Integrationsschübe im großen Wirtschaftsraum Europa. Die Fortentwicklung der Organstruktur der EG/EU, angeführt vom Europäischen Rat als einer ,provisorischen europäischen Regierung“, bot den institutionellen Rückhalt. Trotz der anhaltenden, dabei durchaus unterschiedlich ausgeprägten Bestrebungen der Mitgliedstaaten, die nationale Kernsouveränität nicht aus der Hand zu geben, ist in der Tendenz die Supranationalisierung Europas seit den Römischen Verträgen ständig weitergegangen. Der Binnenmarkt hat mit realisierten Freiheiten im Güter-, Kapital-, Dienstleistungs- und Personenverkehr, mit der Förderung von Freizügigkeit, Niederlassungsfreiheit, Mobilität, mit tendenzieller Harmonisierung der Wirtschaftspolitiken, schließlich mit der gemeinsamen Währung den ökonomischen Großraum unter liberalen Vorzeichen zunehmend strukturiert. Die Rechts- und Wertegemeinschaft gewinnt im „Raum der Freiheit, der Sicherheit und des Rechts“, gefördert durch die Rechtsprechung des Europäischen Gerichtshofs, einen immer festeren Rahmen. Auch die Chance einer Annäherung der Lebensverhältnisse und sozialen Bedingungen in den verschiedenen Teilen der EU ist gegeben, und auf vielen Politikfeldern erfahren die nationalen Innenpolitiken schrittweise eine Angleichung. Die Öffnung der Perspektive einer gemeinsamen Außen- und Sicherheitspolitik nährt die Erwartung, dass das ökonomische Schwergewicht Europa zu einer Politischen Union mit globalem politischem Gewicht mutieren könnte. Die jahrzehntelange Gewohnheit des institutionalisierten Zusammenarbeitens und Zusammenlebens hat die Anwendung militärischer Gewalt zwischen EU-Staaten undenkbar werden lassen. Und die jüngste Finanzkrise könnte durchaus einen - ungeplanten - weiteren Schub in Richtung Supranationalität bringen.

Dass mit dem wirtschaftlichen Großraum auch ein großer Verkehrsraum entstanden ist, war nur folgerichtig. Die Notwendigkeit, im Raum ohne Binnengrenzen Personen und Güter reibungslos zu befördern, hat seit den 1990er Jahren eine gemeinsame Verkehrspolitik, die nach dem EWG-Vertrag schon 1970 hätte verwirklicht sein sollen, geradezu erzwungen. Ziel ist die Herstellung des ,gemeinsamen Verkehrsmarkts“ mit Liberalisierung und Harmonisierung des Verkehrs zu Lande (Straße, Schiene, Binnengewässer), zur See und in der Luft. Ein Programm zum Auf- und Ausbau ,transeuropäischer Netze“ soll der Perspektive eines sich stark ausweitenden Personen- und Güterverkehrs Rechnung tragen, insbesondere im grenzüberschreitenden Verbund nationaler Verkehrsnetze. Es soll den Ausbau der Schienennetze im Hochgeschwindigkeitsbereich und für Alpentunnel fördern sowie nicht zuletzt die Entwicklung von Telematiksystemen und Satellitennavigation (Projekt „Galileo"). Die Dynamik der Gesamtentwicklung mag eine Zahl verdeutlichen: 1958 verfügten die heutigen EU-Länder über 300 Kilometer Autobahnen, heute sind es 52000 Kilometer.

Wir stehen heute also vor einem weitgehend verwirklichten Wirtschafts- und Verkehrsraum Europa, als Ergebnis einer tief greifenden Entwicklung, die mit den Römischen Verträgen 1957 ihren Anfang nahm. Es ist daher durchaus angemessen, den Ort ihrer Unterzeichnung als europäischen Erinnerungsort ersten Ranges einzuordnen, mehr noch als den Uhrensaal des Pariser Quai d'Orsay. Als Chiffre für das europäische Einigungsprojekt insgesamt steht er gewiss in Konkurrenz mit anderen Orten. Da ist Straßburg, seit 1950 Ort 
des Europäischen Parlaments und schon vorher Sitz des Europarats und des Europäischen Gerichtshofs für Menschenrechte - und die Stadt deutsch-französischer Geschichte. Auch Luxemburg hat hohe Symbolträchtigkeit, der Ort der Hohen Behörde der Montanunion und des Europäischen Gerichtshofs, dem in den 1950er Jahren die europäische Hauptstadtrolle sogar förmlich, aber vergeblich, angetragen wurde. Vor allem aber Brüssel, seit 1957 Sitz der Europäischen Kommission und ihrer Vorläufer sowie des Ministerrats, die Stadt, in der sich zunehmend alle europäischen Institutionen konzentrieren, das institutionelle Herz der Europäischen Union, die inzwischen gar nicht mehr heimliche Kapitale. Als geschichtlicher Ort aber, an dem die europäische Einigung eigentlich erst richtig begann, hat der Unterzeichnungsort der Römischen Verträge eine einzigartige Stellung und Würde, vielleicht sogar als Ort einer künftigen identitätsstiftenden Legende.

\section{Literaturhinweise}

40 ans des Traités de Rome, ou la capacité des Traités d'assurer les avancées de la construction européenne./40 years of the Treaties of Rome, or the capacity of the Treaties to advance the European integration processs./40 Jahre Römische Verträge, oder das Vermögen der Verträge, den europäischen Aufbau voranzutreiben./40 anni dei Trattati di Roma, o l'effiacia dei Trattati nell'assicurare I progressi della costruzione europea, Actes du Colloque de Rome, 26-27 mars 1997 sous la direction de la Commission européenne, Bruxelles 1999.

Berliner Erklärung anlässlich des 50. Jahrestages der Unterzeichnung der Römischen Verträge http://europa.eu/50/docs/berlin_declaration_de.pdf (15.6.2011)

Franz Knipping, Rom, 25 März 1957. Die Einigung Europas. München 2004.

Franz KNIPPING, 50 Jahre Römische Verträge. Der Einfluss der Nationalstaaten auf die europäische Integration, in: Andreas Marchetтi/Martin Zimmeк (Hrsg.), Annäherungen an Europa. Beiträge zur deutschen Ratspräsidentschaft 2007. Bonn 2007.

Wilfried Lотн (Hrsg.), Experiencing Europe. 50 Years of European Construction 1957-2007. Baden-Baden 2009.

Enrico SERra (Hg.), Il rilancio dell'Europa e I Trattati di Roma/La relance européenne et les Traités de Rome/The Relaunching of Europe and the Treaties of Rome, Actes du Colloque de Rome 25-28 mars 1987. Bruxelles 1989. 
